\title{
The Implementation of Inspiratory Muscle Training to Enhance Weaning from Mechanical Ventilation: A Systematic Literature Review
}

\author{
Anton Priambodo ${ }^{1,2}$ \\ Henny Suzanna Mediani ${ }^{3}$ \\ Etika Emaliyawati ${ }^{\star}$ (D) \\ Master in Nursing Study Program, \\ Faculty of Nursing, Universitas \\ Padjadjaran, Bandung, Indonesia. \\ 2 Sekolah Tinggi IImu Kesehatan \\ Horizon Karawang, Karawang, \\ Indonesia. \\ 3 Department of Fundamental and \\ Pediatric Nursing, Faculty of \\ Nursing, Universitas Padjadjaran, \\ Bandung, Indonesia. \\ 4 Department of Medical-Surgical, \\ Critical, Emergency, and Disaster \\ Nursing, Faculty of Nursing, \\ Universitas Padjadjaran, Bandung, \\ Indonesia.
}

${ }^{*}$ Correspondence: Etika Emaliyawati Department of Medical-Surgical, Critical, Emergency, and Disaster Nursing, Faculty of Nursing, Universitas Padjadjaran, Bandung, Indonesia.

Phone: (022) 7795596.

Fax: (022) 7795596

Email: etika@unpad.ac.id

Volume 1(1), 16-22

(c) The Author(s) 2022

http://dx.doi.org/10.55048/jpns.v1i1.3

e-ISSN 2827-8100

p-ISSN 2827-8496

Received : December 30, 2021

Revised : January 5, 2022

Accepted : January 6, 2022

Published: January 22, 2022

\section{(i) ()}

This is an Open Access article distributed under the terms of the Creative Commons Attribution-NonCommercial 4.0 International License.

\begin{abstract}
Background: Mechanical ventilation is very important for the world of critical care. However, the use of mechanical ventilation also affected respiratory muscle weakness.

Objective: The purpose of this study was to identify the implementation of Inspiratory muscle training for weaning mechanical ventilation in patients with mechanical ventilation in the Intensive Care Unit.

Design: A systematic literature review study design was used. Data Sources: The databases used are CINAHL, PubMed, MEDLINE, Sage Journal, and ScienceDirect.

Review Methods: Articles published from 2009-2020 with the type of randomized research-controlled trial (RCT) in English. The keywords "Inspiratory Muscle Exercise", "Inspiratory Muscle Training", "weaning", "mechanically ventilated", "critical care", and "intensive care".

Results: From four articles analyzed, we found an effect of inspiratory muscle training on ventilator weaning, which was carried out on therapy five times a week, and some were carried out for seven days for two weeks and increased respiratory muscle strength.

Conclusion: Inspiratory muscle training in mechanically ventilated patients is a nursing intervention that requires evidence-based preparation of guidelines to provide best practice. The optimalization of inspiratory muscles exercise can help the weaning process and be implemented further.
\end{abstract}

Keywords: inspiratory muscle training; weaning; ventilator; intensive care unit

\section{INTRODUCTION}

Mechanical ventilation plays an important role in the world of critical care. The role of mechanical ventilation can be used as a substitute for ventilation in patients with lung disorders (Giusti et al., 2021; Gupte et al., 2022). However, mechanical ventilation can also cause respiratory muscle weakness among patients in the intensive care unit (ICU) (Walsh et al., 2021). With the use of controlled mechanical ventilation for 18-69 hours, there will be disturbances in the diaphragm proteolysis occurs atrophy and respiratory muscle weakness (Bissett et al., 2016).

Priambodo, A., Mediani, H. S., \& Emaliyawati, E. (2022). The Implementation of Inspiratory Muscle Training to Enhance Weaning from Mechanical Ventilation: A Systematic Literature Review. The Journal of Palembang Nursing Studies. 1(1): 16-22. http://dx.doi.org/10.55048/ jpns.v1i1.3 


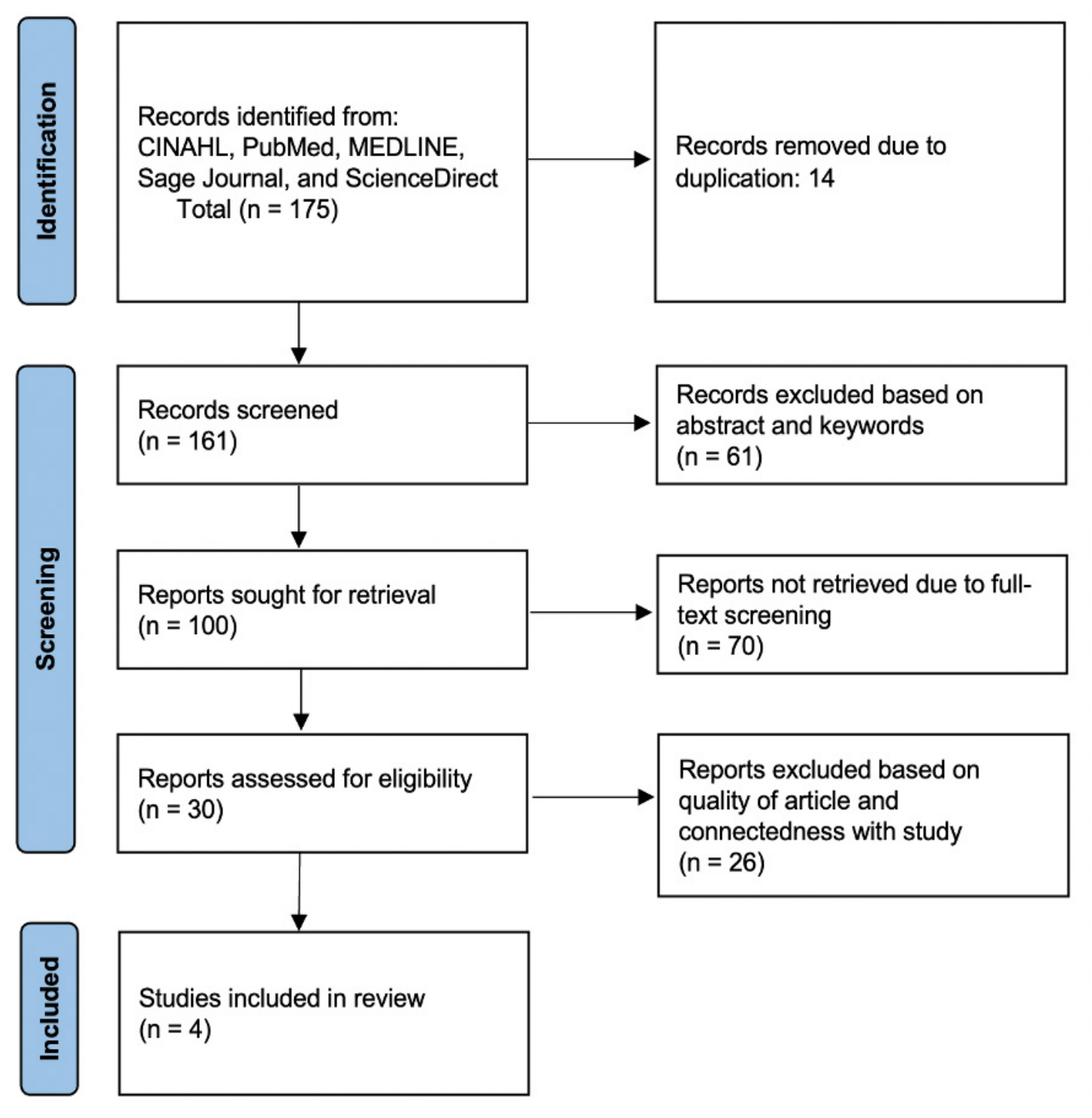

Figure 1. Article Search Strategy

The weakness of the respiratory muscles can contribute to the occurrence of persistent dyspnoea, where the residual physical function disorder will worsen the condition. In addition, if patients use mechanical ventilation, then proteolysis of skeletal muscles and diaphragm occurs, complicating the weaning process (Chiu et al., 2020; Spiesshoefer et al., 2020). The previous studies showed that in patients with ventilators used the strength of the diaphragm significantly affected the process of weaning the patient to breathe independently after the escape of a mechanical ventilator (Bissett et al., 2016). Besides that, the respiratory muscles can be returned to their optimum in several ways, such as by giving an inspiration load and respiratory muscles independent of mechanical ventilation (Condessa et al., 2013). In restoring the respiratory muscle strength, the patient needs to be prepared for the weaning process and not depend on the ventilator. The previous studies related to the implementation of Inspiratory Muscle Training is not reported well. Thus, this literature review can provide new information about inspiratory muscle training to improve the weaning process. In addition, this literature review can provide new insight among nurses and other healthcare teams. Thus, the purpose of this study was to identify the implementation of Inspiratory muscle training for weaning mechanical ventilation in patients with mechanical ventilation in the Intensive Care Unit. 


\section{METHODS}

\section{Study Design}

The study design was a systematic literature review.

\section{Search Methods}

This literature study was compiled from studies published online. A PRISMA methods was used. The search was conducted in June 2020 , with the search criteria covering articles published in English and published from 20092020. The databases used are CINAHL, PubMed, MEDLINE, Sage Journal, and ScienceDirect with the keywords "Inspiratory Muscle Exercise", "Inspiratory Muscle Training", "Weaning", "mechanically ventilated", "critical care", and "intensive care". The first identified articles through keywords were 175 articles. Of the 175 articles screened, 165 were excluded from search results because of titles and/or abstracts, such as research designs that were not relevant to the literature review topic. The next check is the feasibility of full-text articles conducted on 30 articles. The results obtained from the selection process are four articles with the type of RCT that will be carried out in the process literature review (Figure 1).

\section{Inclusion and Exclusion Criteria}

The inclusion criteria were an article in English, a full paper, and an RCT study design. We eliminated the articles out of inclusion criteria and did not focus on inspiratory muscle training.

\section{Data Extraction}

The selection of reviewed articles focused on the type of experimental research, either controlled trial randomized (RCT) or quasiexperimental study. There are still limited RCT studies on Inspiratory muscle training in mechanically ventilated patients in the ICU. Therefore, the selected articles include research on the implementation, comparison, and effectiveness of Inspiratory muscle training on the successful process of ventilator weaning. The review involved adult patients in the ICU who were intubated and mechanically ventilated. The outcome of this review is the effect of Inspiratory muscle training on the success of ventilator weaning.

\section{RESULTS}

Database (CHINAHL, PubMed, MEDLINE,
Sage Journal, and ScienceDirect) with the keywords "Inspiratory Muscle Exercise", "Inspiratory Muscle Training", "Weaning", "mechanically ventilated", "critical care", and "intensive care". The first identified articles through keywords were 175 articles. Of the 175 articles screened, 165 were excluded from search results because of titles and/ or abstracts, such as research designs that were not relevant to the topic of the literature review. From the article that describes the effectiveness of respiratory muscle training, it was found that there was an effect of respiratory muscle training on ventilator weaning, which was carried out on therapy 5 times a week, and some were carried out for 7 days for 2 weeks, but in other studies there are also which has no effect on weaning time, only inspiratory muscle therapy can increase respiratory muscle strength, where if the respiratory muscles can work optimally it will help the weaning process (Table 1).

\section{DISCUSSION}

This literature review consists of articles that have heterogeneity in therapy-respiratory muscle exercise in patients on mechanical ventilation to achieve weaning on mechanical ventilation. The previous study showed that respondents who completed two weeks of IMT had a more significant increase in respiratory muscle strength than those who did not. This is related to the previous study that ventilation mechanics increase in strength within two weeks of IMT (Bissett et al., 2016). The increase in respiratory muscle strength can be caused by the effects of exercise such as IMT and the MIP (Maximal Inspiration Pressure) test manoeuvre (Cabrita et al., 2021; Han et al., 2020; Tanriverdi et al., 2021)

However, atrophy may occur quickly in ICU patients, especially in skeletal muscle and respiratory muscles (Spiesshoefer et al., 2020), if the patient is attached to a mechanical ventilator for more than five days. With an adequate training stimulus, the possibility of atrophy in the respiratory muscles will decrease in a relatively short period of time, so that it will help the ventilator weaning process. IMT can be considered an effective strategy to reverse some of the common inspiratory muscle weakness after prolonged use of mechanical ventilation and improve the quality of life in these patients with only two weeks of training (Hoffman et al., 2018).

Priambodo, A., Mediani, H. S., \& Emaliyawati, E. (2022). The Implementation of Inspiratory Muscle Training to Enhance Weaning from Mechanical Ventilation: A Systematic Literature Review. The Journal of Palembang Nursing Studies. 1(1): 16-22. http://dx.doi.org/10.55048/ jpns.v111.3 


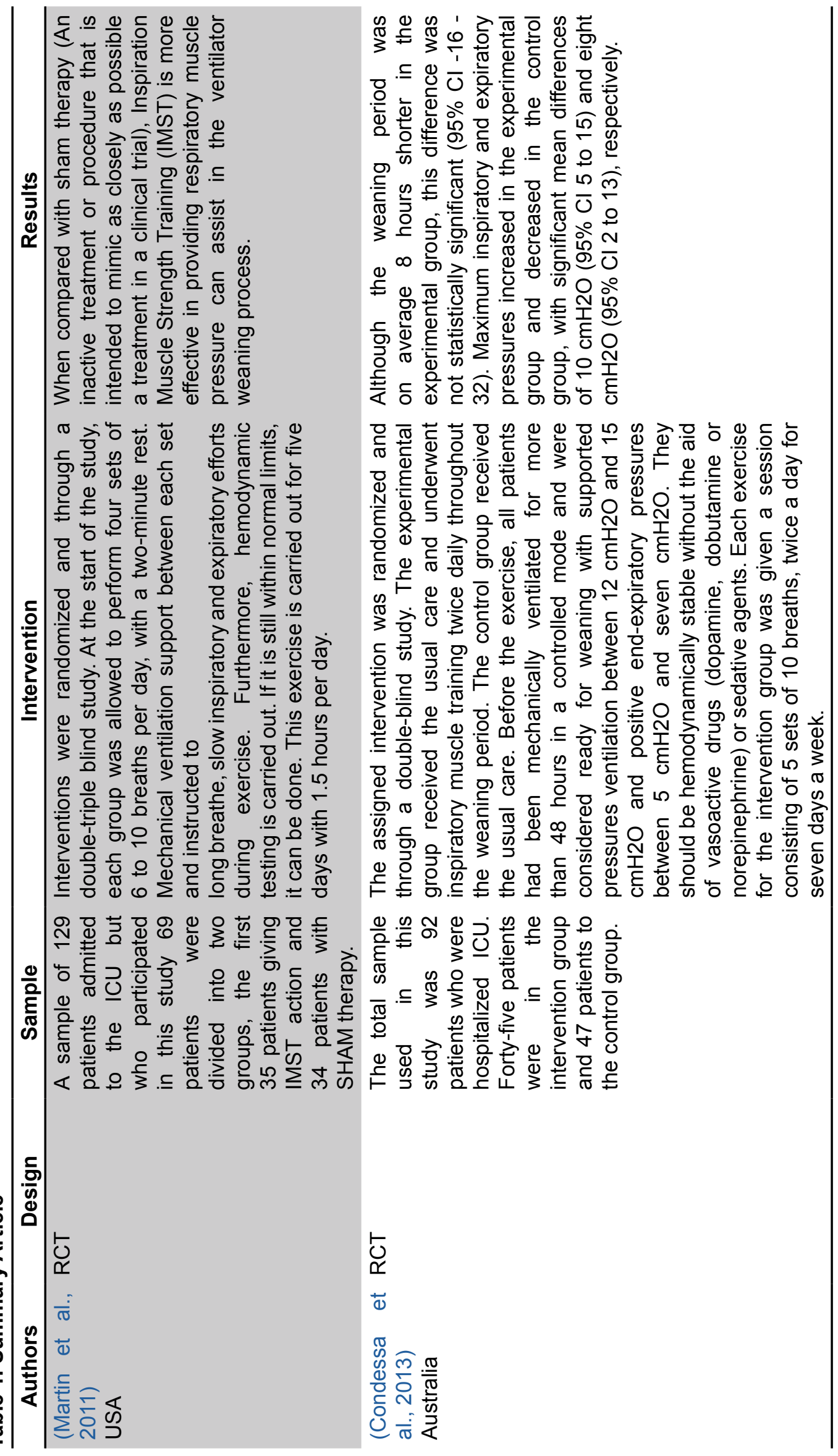

Priambodo, A., Mediani, H. S., \& Emaliyawati, E. (2022). The Implementation of Inspiratory Muscle Training to Enhance Weaning from Mechanical Ventilation: A Systematic Literature Review. The Journal of Palembang Nursing Studies. 1(1): 16-22. http://dx.doi.org/10.55048/ jpns.v1i1.3 


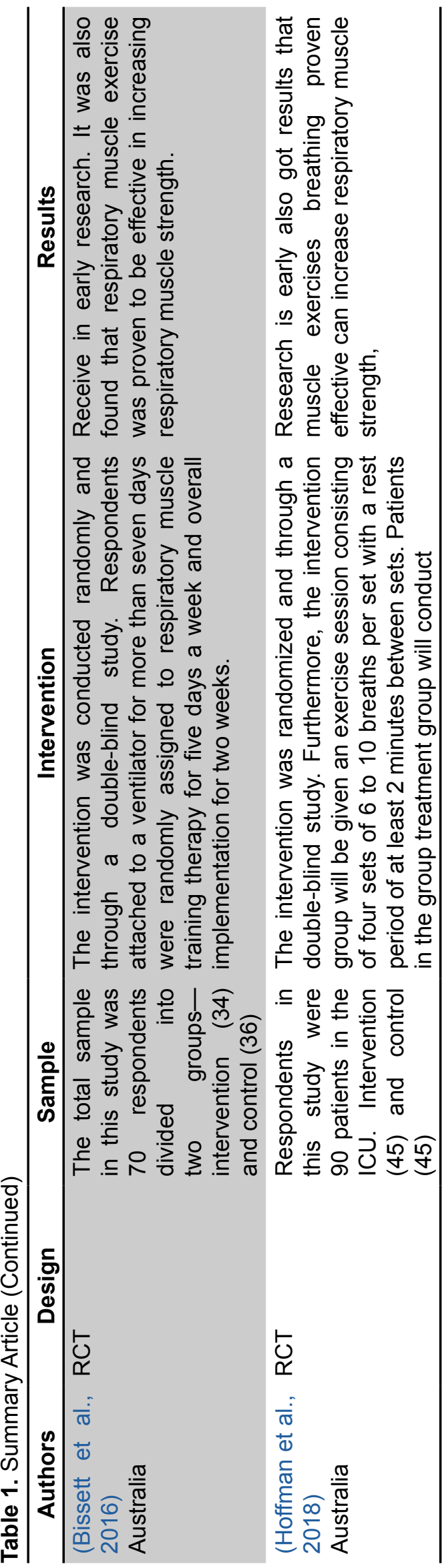

Inspiratory muscle training during the weaning period can increase maximal inspiratory and expiratory pressures and tidal volume, although it does not significantly reduce the weaning period (Bissett et al., 2012; Condessa et al., 2013). For example, inspiratory muscle training was performed by adjusting the ventilator pressure's trigger sensitivity to $20 \%$ of the maximal inspiratory pressure and increased for 5 minutes in each session until it reached 30 minutes. After that, the pressure will increase by $10 \%$ of the initial maximum inspiratory pressure to a maximum of $40 \%$ of the maximum inspiratory pressure (Martin et al., 2011).

Using a threshold device with an initial load of $30 \%$ of the maximum inspiratory pressure can increase by $10 \%$ every day for 5 minutes (Boswell-Ruys et al., 2020). A threshold device set at the highest tolerable pressure is between 7 and $12 \mathrm{cmH} 2 \mathrm{O}$ (Martin et al., 2011). A previous study showed a maximal inspiratory pressure could be evaluated before each session, and training load was improved at $40 \%$ of this value, which equates to an average of $13 \mathrm{cmH} 2 \mathrm{O}$ initially (Condessa et al., 2013). Therefore, the initial load will be higher than in other studies in this area. It may also contribute to the significant increase in maximal inspiratory pressure, roughly the same as the most significant increase seen in other studies (Syabbalo, 1998). However, the weaning period did not differ significantly between the experimental and control groups. Therefore, inspiratory muscle training with a threshold device may be adequate for increasing respiratory muscle strength and tidal volume in patients receiving mechanical ventilation.

\section{CONCLUSION}

Inspiratory muscle training in mechanically ventilated patients is an intervention nursing that requires evidence-based preparation of guidelines to provide best practice. Without evidence-based practice, nurses in critical nursing areas will assist patients in the mechanical ventilation weaning process. This study supports that the perception of inspiratory effort and respiratory drive is disproportionate to inspiratory muscle strength and may help explain why increased MIP (Maximal Inspiration Pressure) contributes to weaning.

\section{Declaration of Interest}

None

Priambodo, A., Mediani, H. S., \& Emaliyawati, E. (2022). The Implementation of Inspiratory Muscle Training to Enhance Weaning from Mechanical Ventilation: A Systematic Literature Review. The Journal of Palembang Nursing Studies. 1(1): 16-22. http://dx.doi.org/10.55048/ jpns.v1i1.3 


\section{Acknowledgment}

None

\section{Funding}

None

\section{Data Availability}

The datasets generated during and/or analyzed during the current study are available from the corresponding author on reasonable request.

\section{REFERENCES}

Bissett, B., Leditschke, I. A., Paratz, J. D., \& Boots, R. J. (2012). Respiratory dysfunction in ventilated patients: can inspiratory muscle training help? Anaesth Intensive Care, 40(2), 236-246. https:// doi.org/10.1177/0310057X1204000205

Bissett, B. M., Leditschke, I. A., Neeman, T., Boots, R., \& Paratz, J. (2016). Inspiratory muscle training to enhance recovery from mechanical ventilation: a randomised trial. Thorax, 71(9), 812. https://doi. org/10.1136/thoraxjnl-2016-208279

Boswell-Ruys, C. L., Lewis, C. R. H., Wijeysuriya, N. S., McBain, R. A., Lee, B. B., McKenzie, D. K., Gandevia, S. C., \& Butler, J. E. (2020). Impact of respiratory muscle training on respiratory muscle strength, respiratory function and quality of life in individuals with tetraplegia: a randomised clinical trial. Thorax, 75(3), 279. https://doi.org/10.1136/ thoraxjnl-2019-213917

Cabrita, B., Dias, S., Fernandes, A. L., Correia, S., Ferreira, J., \& Simao, P. (2021). Inspiratory muscle training in neuromuscular patients: Assessing the benefits of a novel protocol. J Back Musculoskelet Rehabil, 34(4), 537-543. https://doi.org/10.3233/BMR-200141

Chiu, K. L., Hsieh, P. C., Wu, C. W., Tzeng, I. S., Wu, Y. K., \& Lan, C. C. (2020). Exercise training increases respiratory muscle strength and exercise capacity in patients with chronic obstructive pulmonary disease and respiratory muscle weakness. Heart Lung, 49(5), 556-563. https://doi.org/10.1016/j. hrtlng.2020.03.005

Condessa, R. L., Brauner, J. S., Saul, A. L., Baptista, M., Silva, A. C. T., \& Vieira, S. R. R. (2013). Inspiratory muscle training did not accelerate weaning from mechanical ventilation but did improve tidal volume and maximal respiratory pressures: a randomised trial. Journal of physiotherapy, 59(2), 101-107. https:// doi.org/10.1016/S1836-9553(13)701620

Giusti, S., Chazin, S., Vaitla, P., Atiemo, K., Atari, M., Paramesh, A., Jeon, H., Torres-Ortiz, A., Thimmisetty, R., \& Garces, J. (2021). Observational Study of the Clinical Characteristics and ShortTerm Outcomes of Kidney Transplant Recipients Diagnosed With COVID-19 Infection (SARS-CoV-2) Requiring Hospitalization in New Orleans. Ochsner J, 21(4), 329-334. https://doi. org/10.31486/toj.21.0008

Gupte, V., Hegde, R., Sawant, S., Kalathingal, K., Jadhav, S., Malabade, R., \& Gogtay, J. (2022). Safety and clinical outcomes of remdesivir in hospitalised COVID-19 patients: a retrospective analysis of active surveillance database. BMC Infect Dis, 22(1), 1. https://doi.org/10.1186/ s12879-021-07004-8

Han, Y. J., Lee, J., Sohn, D. G., Park, G. Y., Kim, Y., Park, H. Y., Jung, S. A., \& Im, S. (2020). Cut-off Values of the Respiratory Muscle Power and Peak Cough Flow in Post-Stroke Dysphagia. Medicina (Kaunas), 56(12). https://doi. org/10.3390/medicina56120635

Hoffman, M., Van Hollebeke, M., Clerckx, B., Muller, J., Louvaris, Z., Gosselink, R., Hermans, G., \& Langer, D. (2018). Can inspiratory muscle training improve weaning outcomes in difficult to wean patients? A protocol for a randomised controlled trial (IMweanT study). BMJ OPEN, 8(6), e021091. https://doi. org/10.1136/bmjopen-2017-021091

Martin, A. D., Smith, B. K., Davenport, P. D., Harman, E., Gonzalez-Rothi, R. J., Baz, M., Layon, A. J., Banner, M. J., Caruso, L. J., Deoghare, H., Huang, T.-T., \& Gabrielli, A. (2011). Inspiratory muscle strength training improves weaning outcome in failure to wean patients: a randomized trial. Critical Care, 15(2), R84. https://doi.org/10.1186/cc10081

Spiesshoefer, J., Orwat, S., Henke, C., Kabitz, H. J., Katsianos, S., Borrelli, C., Baumgartner, H., Nofer, J. R., Spieker, M., Bengel, P., Giannoni, A., Dreher, M., Boentert, M., \& Diller, G. P. (2020). Inspiratory muscle dysfunction and

Priambodo, A., Mediani, H. S., \& Emaliyawati, E. (2022). The Implementation of Inspiratory Muscle Training to Enhance Weaning from Mechanical Ventilation: A Systematic Literature Review. The Journal of Palembang Nursing Studies. 1(1): 16-22. http://dx.doi.org/10.55048/ jpns.v1i1.3 
restrictive lung function impairment in congenital heart disease: Association with immune inflammatory response and exercise intolerance. Int J Cardiol, 318, 45-51. https://doi.org/10.1016/j. ijcard.2020.06.055

Syabbalo, N. (1998). Respiratory muscle function in patients with neuromuscular disorders and cardiopulmonary diseases. Int J Clin Pract, 52(5), 319-329. https:// www.ncbi.nlm.nih.gov/pubmed/9796564

Tanriverdi, A., Kahraman, B. O., Ozsoy, I., Ozpelit, E., \& Savci, S. (2021). Acute effects of inspiratory muscle training at different intensities in healthy young people. Ir J Med Sci, 190(2), 577-585. https://doi.org/10.1007/s11845-02002353-w

Walsh, L. J., Deasy, K. F., Gomez, F., O’Sullivan, E., Eustace, J., Ryan, A. M., \& Murphy, D. M. (2021). Use of non-invasive ventilation in motor neuron disease - a retrospective cohort analysis. Chron Respir Dis, 18, 14799731211063886. https://doi. org/10.1177/14799731211063886

Priambodo, A., Mediani, H. S., \& Emaliyawati, E. (2022). The Implementation of Inspiratory Muscle Training to Enhance Weaning from Mechanical Ventilation: A Systematic Literature Review. The Journal of Palembang Nursing Studies. 1(1): 16-22. http://dx.doi.org/10.55048/ jpns.v1i1.3 